\title{
EVALUATION OF VENTRICULOPERITONEAL SHUNT SURGERY THROUGH CONVENTIONAL AND VENTRICULOSCOPIC TECHNIQUE
}

\author{
Anurag Sahu1, Neeraj Shende2, Kulwant Singh ${ }^{3}$, Vivek Sharma ${ }^{4}$ \\ ${ }^{1}$ Senior Resident, Department of Neurosurgery, Institute of Medical Sciences, Banaras Hindu University, Varanasi. \\ ${ }^{2}$ Senior Resident, Department of Neurosurgery, Institute of Medical Sciences, Banaras Hindu University, Varanasi. \\ ${ }^{3}$ Associate Professor, Department of Neurosurgery, Institute of Medical Sciences, Banaras Hindu University, Varanasi. \\ ${ }^{4}$ Professor, Department of Neurosurgery, Institute of Medical Sciences, Banaras Hindu University, Varanasi.
}

\section{ABSTRACT}

\section{BACKGROUND}

Aims \& Objective- To compare the position of ventricular end between conventional and Ventriculoscope guided technique.

\section{MATERIALS AND METHODS}

Patients undergone ventriculo-peritoneal shunt from August 2013 to September 2014 were included in this study. Total forty cases were included and divided into two groups. The first group comprised of shunt surgery using conventional method of shunt placement as per anatomical landmarks. The second group had proximal end placement under direct endoscopic (Ventriculoscope) visualization. The relationship between proximal ventricular catheter position and shunt revision rate was evaluated using postoperative computed tomography by a 3-point scale: 1) Grade I; Position of tip is anterior to foramina of Monro, free-floating in cerebrospinal fluid, 2) Grade II; touching choroid plexus or ventricular wall, tip is posterior to foramina of Monro 3) Grade III; tip within parenchyma, crossing to opposite side.

\section{RESULTS}

Forty patients were considered, and divided into two groups: 1 . Ventricular end insertion through conventional technique, 2. Ventriculoscope guided ventricular end insertion. There were 20 patients in each group. In conventionally treated study group, 12 patients $(60 \%)$ revealed grade 1 placement of ventricular end, 6 patients $(30 \%)$ had grade 2 , and 2 patients $(10 \%)$ showed grade 3 in post-operative CT scan. endoscopic study group had 19 patients (95\%) in grade 1 placement and 1 patients (5\%) in grade 2 while none was in grade 3 on post-operative CT scan. Infection rate was same in both groups.

\section{CONCLUSION}

The use of navigated system in routine shunt surgery can eliminate poor shunt placement resulting in a dramatic reduction in shunt revision rates.

\section{KEYWORDS}

Ventriculoscope, Hydrocephalus, Ventriculoperitoneal Shunt.

HOW TO CITE THIS ARTICLE: Sahu A, Shende N, Singh K, et al. Evaluation of ventriculoperitoneal shunt surgery through conventional and ventriculoscopic technique. J. Evolution Med. Dent. Sci. 2017;6(2):136-140, DOI: 10.14260/Jemds/2017/33

\section{BACKGROUND}

Babies born with hydrocephalus (Congenital) and adults or children who develop hydrocephalus (Acquired) usually need prompt treatment to reduce the pressure on their brain. If hydrocephalus isn't treated, the increase in pressure will cause brain damage.

Dandy and Blackfan ${ }^{1}$ (1914) proved the existence of two distinct types of hydrocephalus.

1. The obstructive (or non-communicating) type.

2. The non-obstructive (or communicating) type.

They established that the cause of non-communicating (Obstructive) hydrocephalus was the inability of the cerebrospinal fluid to escape from the obstructed ventricles

Financial or Other, Competing Interest: None.

Submission 14-10-2016, Peer Review 22-12-2016,

Acceptance 29-12-2016, Published 05-01-2017.

Corresponding Author:

Dr. Anurag Sahu,

Senior Resident,

Department of Neurosurgery,

Institute of Medical Sciences,

Banaras Hindu University, Varanasi.

E-mail: anuragsahu25@rediffmail.com

DOI: $10.14260 /$ jemds $/ 2017 / 33$

\section{(c) $(1)$}

to the subarachnoid system where it could be absorbed by natural processes; and that the cause of communicating (Nonobstructive) hydrocephalus was impaired absorption of the cerebrospinal fluid after it has reached the subarachnoid system because of congenital mal-development of the subarachnoid spaces or their obliteration by postinflammatory adhesions.

While Ventriculoperitoneal shunt is certainly not the most glamorous neurosurgical operation, but it is one of the basic neurosurgical procedure, and also has the highest failure rate. It has a relatively high complication rate and is probably the most common operation which has to be redone for either malfunction or infection. Clearly, one of the best ways of managing shunt problems is avoiding them in the first place. The most common cause of ventricular shunt malfunction is proximal shunt occlusion, with the ventricular catheter frequently blocked by choroid plexus and/or brain parenchyma. Accurate placement of ventricular catheter depends on both proper insertion trajectory and proper catheter tip positioning vis-a-vis ventricular configuration. Conditions such as small ventricle or slit ventricle syndrome may increase the likelihood of shunt misplacement. In such cases, the introduction of a new ventricular catheter may turn out to be very difficult, including a significant risk of 
complications. Mechanical malfunction and infection are the most significant problems associated with shunts for the treatment of hydrocephalus. Above all, a significant proportion of shunt failure was due to obstruction of the ventricular catheter, and accurate placement of the shunt catheter is highly important to reduce the incidence of shunt malfunction. The standard conventional technique, which relies on surface anatomic landmarks and the surgeon's sense of spatial orientation, is not consistently accurate in the positioning the ventricular shunt catheter. Besides intraoperative confirmation of position of ventricular end is not possible. Ventricular anatomy might provide difficult circumstances for accurate placement of cerebro-spinal fluid (CSF) catheters for ventricular peritoneal shunting. The introduction of the ventriculoscope has provided intraoperative intraventricular direct confirmation of position of ventricular end of shunt. Besides giving opportunity for intraoperative evaluation of intraventricular anatomy and subsequent therapeutic and diagnostic intervention could be done in same sitting. The ventriculoscope has not produced any complication and also no damage to vascular structure. The clear image allows the proper placement of the ventricular catheter away from the choroid plexus of the third ventricle, and the coagulation of any choroid plexus if needed.

The present study was a done to evaluate and compare the conventional method of ventriculoperitoneal shunt insertion with ventriculoscopic Guided shunt surgery.

\section{MATERIALS AND METHODS}

All the patients of CT proven Hydrocephalus who have been managed prospectively in neurosurgery department will be included in study. Study comprised of total 40 cases along with 20 cases of each conventional shunt insertion and ventriculoscopic guided shunt insertion.

The surgical procedure is as follows: The valve type and catheter were used depending on the surgeon's preference. In the standard group, skin incision and trephination of the cranium was done by using anatomical landmarks like Kocher's point or Frazier's point. Ventricular catheter length was determined based on surgeon's measurement using brain CT imaging. The entry point was localized at the junction of the plane approximately $2 \mathrm{~cm}$ lateral to the midline in the midpupillary line intersecting the line drawn from superior aspect of pinna. The target point was determined and set at the foramen of Monro. The patient was then prepared and draped in the usual sterile fashion appropriate for a ventriculoperitoneal shunt placement. A comma-shaped incision was made around the entry point and a subcutaneous pocket was prepared for the shunt valve. Distal tunnelling from the entry point passing the retroauricular region to the paraumbilical abdomen was performed in a standard fashion. A burr hole through the skull was made following the predefined trajectory. The duramater was cauterized and incised. The ventricular catheter was cut in fish mouth technique towards it blind ended distal end. The ventricular catheter was worn over the ventriculoscope. Then, introduce a catheter over a shunt insertion trajectory, insert catheter parallel to skull base aiming to either middle of forehead or ipsilateral medial canthus of eye The entry point previously located on the skin was converted to the pivoting point of the instrument holder consequently, the neuronavigation workstation showed the trajectory length. A ventricular catheter was marked at the appropriate length. A custom made catheter guidance tube (Shaft diameter $3.1 \mathrm{~mm}$ ) was inserted in the instrument holder. A Ventriculoscope was used to direct the catheter straight over the shunt trajectory into the ventricle, Cerebro Spinal Fluid egression was observed and the ventriculoscope was advanced further under ventriculoscopic vision to just ahead of foramen of Monro while visualising the intraventricular land marks. The ventriculoscope was then withdrawn. The catheter was then secured to the valve. Before introducing the distal drain tip in the abdomen, flow patency of the shunt was ascertained. Wound closure was done in a standard manner.

\section{Inclusion and Exclusion Criteria}

1. All cases of CT proven hydrocephalus requiring shunt were included in the study.

2. Acute phase of bacterial and tubercular meningitis presenting as hydrocephalus were excluded from study.

3. Cases were randomly subjected to conventional shunt insertion and ventriculoscopic and navigation assisted shunt insertion.

4. On $5^{\text {th }}$ post-operative day, NCCT head of all patients was done and position of proximal catheter position (Shunt tip) was evaluated using postoperative computed tomography by a 3-point scale.

Grade I-Optimal position free-floating in cerebrospinal fluid, Grade II-Touching choroid or ventricular wall, Grade III-Tip within parenchyma.

\section{RESULTS}

In this study patients were divided in two study groups randomly one group was subjected to conventional method and other group received ventriculoscopic assisted ventriculoperitoneal shunt surgery. The comparative study of conventional, and ventriculoscope assisted shunt surgery revealed following observations.

\section{CSF Finding}

Pre-operative CSF sample revealed no abnormality in 27 cases, and 10 had tubercular finding while 3 cases had pyogenic meningitis.

\begin{tabular}{|c|c|c|}
\hline Finding & No. of cases & percent \\
\hline Normal & 27 & 67.5 \\
\hline Tubercular & 10 & 25 \\
\hline Pyogenic & 3 & 7.5 \\
\hline \multicolumn{2}{|c|}{ Table 1. Pre-operative cerebrospinal fluid analysis } \\
\hline
\end{tabular}

\section{Grades of Proximal Tip Position on Post-Operative Scan}

Proximal catheter position was graded on postoperative brain CT imaging on each case. All patients were graded according the following 3-point scale developed for this study.

\begin{tabular}{|c|c|c|}
\hline & Conventional & Ventriculoscopic \\
\hline Grade I & $12(60 \%)$ & $19(95 \%)$ \\
\hline Grade II & $6(30 \%)$ & $1(5 \%)$ \\
\hline Grade III & $2(10 \%)$ & $0(0 \%)$ \\
\hline \multicolumn{2}{|c|}{ Table 2. Comparison of conventional and } \\
ventriculoscopic (Grades of proximal catheter \\
tip on post-operative CT scan in each study group) \\
\hline
\end{tabular}




\section{Complications}

In present study of 40 cases, five patients had shunt infection in post-operative period and required removal of shunt. Two cases of conventionally placed shunt (Grade 3) did not function well due to proximal obstruction and required shunt revision during same stay in hospital. Among 40 cases of this study, four cases had finding of over drainage.

\begin{tabular}{|c|c|c|c|}
\hline Sl. No. & $\begin{array}{c}\text { Conventional } \\
\text { Shunt } \\
\text { Surgery }\end{array}$ & $\begin{array}{c}\text { Ventriculoscope } \\
\text { Guided Shunt } \\
\text { Surgery }\end{array}$ & $\mathbf{\%}$ \\
\hline 1. Infection & 2 & 3 & 8.3 \\
\hline 2. Obstruction & 2 & 2 & 6.66 \\
\hline $\begin{array}{c}\text { 3. Over- } \\
\text { Drainage }\end{array}$ & 2 & 1 & 5 \\
\hline Malposition & 2 & 0 & 3.33 \\
\hline \multicolumn{2}{|r|}{ Table 3. Complication of shunt surgeries } \\
\hline
\end{tabular}

This study has several statistical limitations. The number of the samples was too small to represent the population of shunt patients. It was necessary to consider other factors that might have influenced the results. However, we tried to include patients who had various factors as possible. All factors except catheter tip position were already proven to have no statistical significance by using multiple regression analysis.

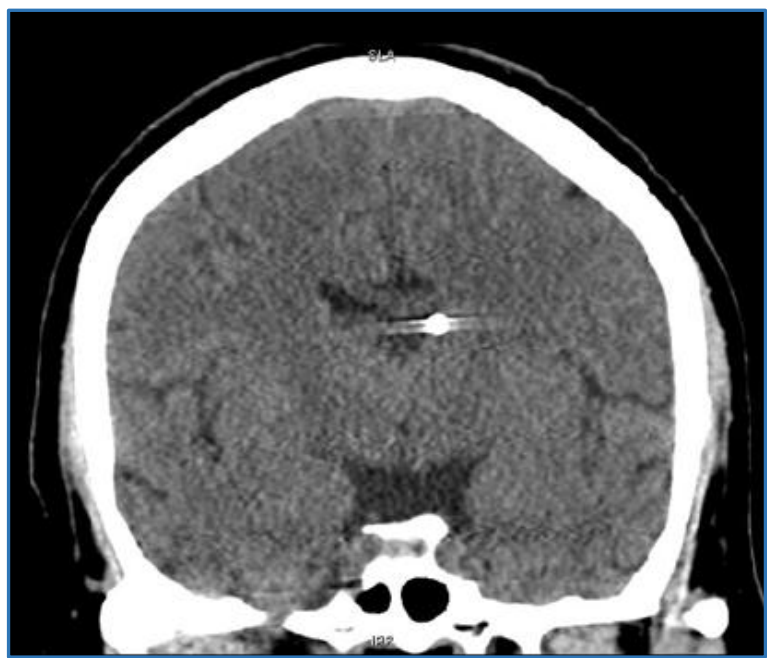

Figure 1. Post-operative CT scan showing position of proximal end (Coronal cut)

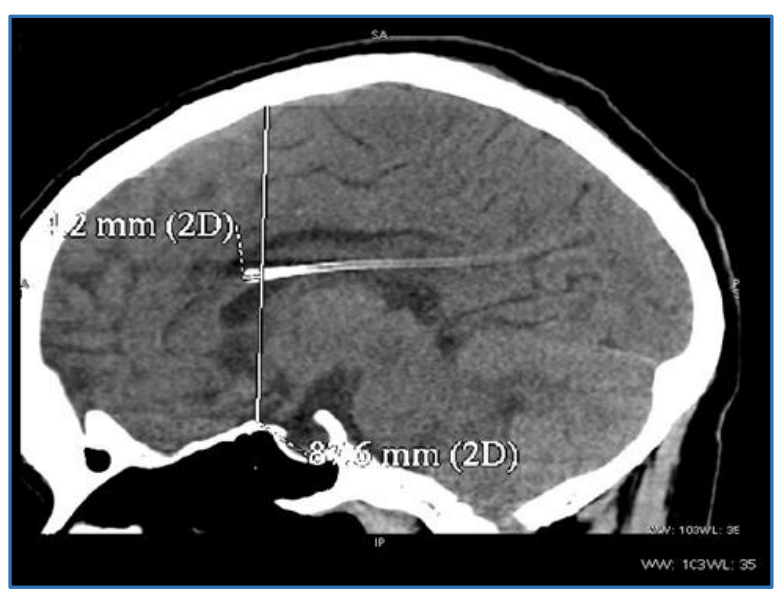

Figure 2. Post-operative CT scan showing position of proximal end (sagittal cut)

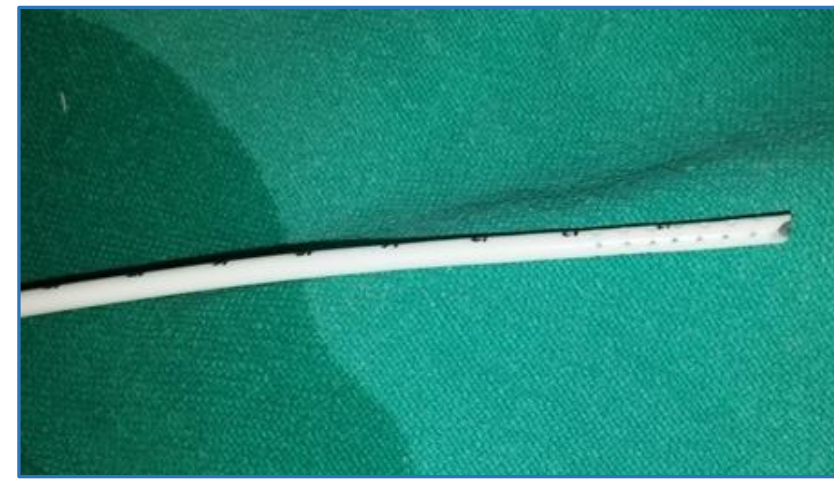

Figure 3. Ventricular end of Shunt with fish mouth cut for ventriculoscope

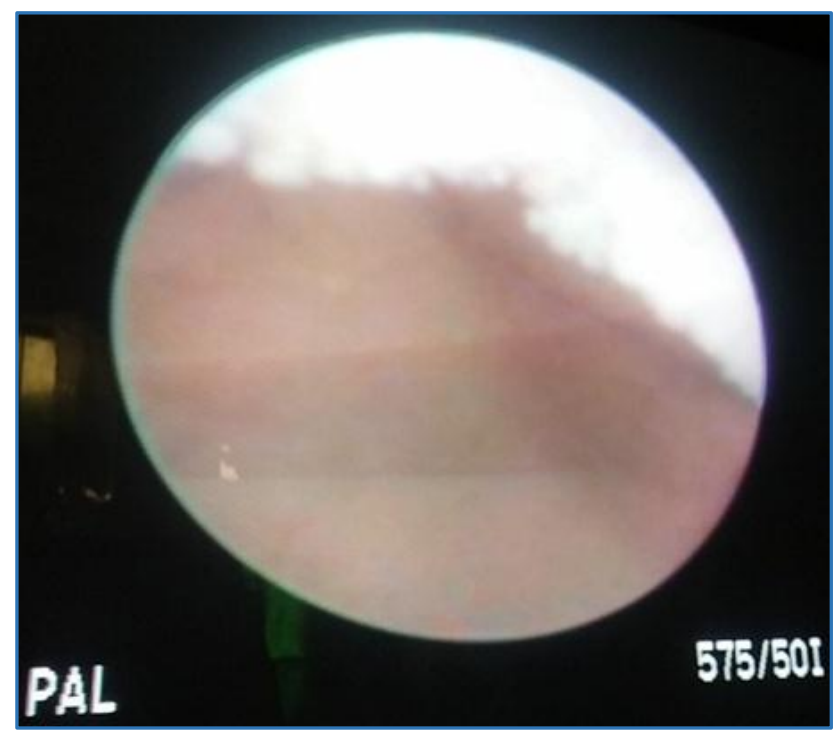

Figure 4. Ventriculoscopic view of choroid plexus

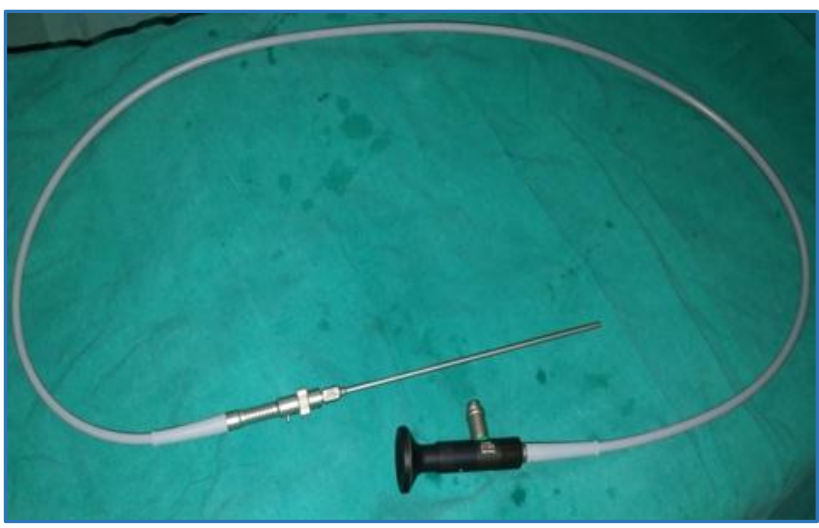

Figure 5. Ventriculoscope

\section{DISCUSSION}

The position of the ventricular shunt catheter is by far most important factor in the long-term patency of a cerebrospinal fluid shunt. However, standard technique, which relies on surface anatomic landmarks and the surgeons sense of spatial orientation, is not consistently accurate in the positioning the ventricular shunt catheter.

Tai Hyoung Cho et $\mathrm{al}^{2}$ found proximal cerebrospinal fluid shunt malfunction due to ventricular catheter occlusion remained the most common source of the shunt problem. The position of the hole-bearing segment of the catheter affects the 
long-term patency of the ventricular catheter of a cerebrospinal fluid shunt. Placement of this segment near the choroid plexus or injured ependyma increases the probability of obstruction.

Yamada SM et $\mathrm{al}^{3}$ found that long-term maintenance of ventriculoperitoneal (VP) shunt function depends on the correct placement of the catheter tip in the lateral ventricle.

The result of present study was comparable to literature. Pang and Grabb ${ }^{4}$ reported that long-term patency of the ventricular catheter of a cerebrospinal fluid shunt depends on the positioning of the hole-bearing segment of the catheter. Placement of this segment near the choroid plexus or injured ependyma increases the probability of obstruction. Proper positioning for a coronal shunt in turn depends on the ventricular catheter length and target coordinates. The authors describe a method of calculating the catheter length based on bone landmarks on skull radiographs, and a technique for accurate ventricular catheter placement using free-hand passage guided by simple stereotactic coordinates based on visible and palpable surface anatomy. The insertion trajectory is aligned with the coronal obliquity of the lateral ventricle so that, even with slit ventricles, the entire holebearing segment of the catheter can be reliably situated within the anterior horn. The predetermined catheter length also fixes the tip at the foramen of Monro, away from the choroid plexus and injured ependyma. (Pang D. et al, 1994)

In 1990 Bierbrauer KS et al. ${ }^{5}$ described most common cause of ventricular shunt malfunction as proximal shunt occlusion. (Bierbrauer KS et al, 1990) while the ventricular catheter frequently blocked by choroid plexus and/or brain parenchyma.

Kestle JR also found that the most common cause of shunt malfunction was ventricular catheter obstruction. (Kestle J.R. et al 2003)6 SekharLN recommended to keep the catheter patent and open, placement of the catheter away from the choroid plexus. This is based on the observation that the tissues in proximity to the catheter tip may grow into or obstruct the inlet holes. Histological studies confirmed that the majority of tissue debris in obstructed ventricular catheters was choroid plexus and ependyma. Injury to these structures may induce inflammatory reaction to a foreign body, which may result in infiltration of glial tissues and inflammatory cells around the shunt catheter tip. (Shekhar L.N. et al 1982) ${ }^{7} \mathrm{CSF}$ shunt valve trials done by Tuli s et al have demonstrated that the catheter tip environment was the most predictive factor for shunt failure. Catheters embedded in brain tissue had the highest failure rate, followed by catheter tips touching brain, as compared with a catheter tip surrounded by CSF. (Tuli S. et al 1999) ${ }^{8}$ Considering all these factors, the optimal position of the ventricular catheter is away from the choroid plexus, and the side holes of proximal inlet segment must not be buried in brain tissue or be in direct contact with injured ependyma. To achieve these goals, the point just in front of the foramen of Monro should be an optimal position for the ventricular catheter tip as changing of the size or shape of the ventricle after surgery does not affect this position.

Lazareff JA 1998, also found that proximal catheter obstruction was known to be the most common cause of malfunctioning of ventriculoperitoneal (VP) shunt followed by infection and disconnection. (Lazareff J.A. et al 1998) ${ }^{9}$ Azeem SS 2007, described that the optimal position of the catheter tip was focused to reduce potential occlusion of proximal catheter by ventricular parenchyma or choroidal tissues. (Azeem S.S. et al 2007) ${ }^{10}$ Many studies including Tuli et al have shown that a ventricular catheter tip surrounded by cerebrospinal fluid (CSF) could decrease the risk of shunt failure. (Tuli S. et al 19998, Wan K.R. et al 201111)

Above all, a significant proportion of shunt failures was due to obstruction of the ventricular catheter, and accurate placement of the shunt catheter was highly important to reduce the incidence of shunt malfunction. (Nayoung Jung et al 2013) ${ }^{12}$

Stephen Price ${ }^{13}$ studied 187 patients and reported that ventricular catheter blockage was the commonest indication for shunt revision. Placement of a ventricular catheter in a region free of choroid plexus prolongs catheter survival. Positioning the tip of the ventricular catheter in the ipsilateral frontal horn anterior to the foramen of Monro is commonly considered the optimum position and is the standard position for catheter placement. Yet ventricular catheter placement is performed blindly and often misses the optimum position. This study was aimed to see how accurate we were in the placement of ventricular catheters and saw whether the good positioning could reduce the rate of shunt revision. One hundred eighty seven catheters were placed in 184 patients. Post-operative imaging was available on 139 patients. Frontal catheters were adequately placed in $67 \%$ of cases; occipital catheters were adequate in $52 \%$. Frontal catheters were frequently too long, whereas occipital catheters commonly crossed the midline. Forty three percent of the burr holes were incorrectly positioned; this might improve with experience. When the burr hole was too lateral, the catheter position was inadequate in $90 \%$ cases. The revision rate for inadequately placed occipital catheters was far higher than adequately placed catheters ( $54 \%$ versus $15 \%$ at 140 weeks), yet there was no difference for the frontal catheters $(50 \%$ vs. $44 \%$ at 140 weeks). Occipital catheters are more difficult to place adequately than frontal catheters. The accuracy of placement of frontal catheters could improve if the depth of insertion could be better controlled. Occipital catheter placement is poor largely due to problems in placing the burr hole. The position of occipital catheters is more critical to shunt survival than frontal catheters. (Stephen Price et al 2006)

Accurate placement of ventricular catheter depends on both proper insertion trajectory and proper catheter tip positioning vis-a-vis ventricular configuration. Conditions such as small ventricles or slit ventricle syndrome may increase the likelihood of shunt misplacement. In such cases, the introduction of a new ventricular catheter may turn out to be very difficult, including a significant risk of complications.

It is debatable whether this currently expensive image guided neuronavigation system should be adopted for simple shunt procedure, but the uncertainty of the ventricular catheter position and the known failure rate of conventional shunt technique are also unacceptable. The study of Yong Bae Kim, Jae Whan Lee, Kyu Sung Lee et al. demonstrated that use of a frameless neuronavigation system was the ideal tool for optimal placement of the ventricular catheter. (Yong Bae Kim et al 2006). ${ }^{14}$

This study has demonstrated that neuronavigation in the placement of ventricular catheter assures optimal catheter position within ventricles, reducing incidence of revision. 


\section{CONCLUSION}

Frameless navigation is a valuable tool to optimize the trajectory and final position of shunt catheter in the ventricle. Neuronavigation has its own limiting factor like its cost and limited availability. Mechanical malfunction and infection are the most significant problems associated with shunts for the treatment of hydrocephalus. Above all, a significant proportion of shunt failure was due to obstruction of the ventricular catheter, and accurate placement of the shunt catheter is highly important to reduce the incidence of shunt malfunction. In conclusion, the use of navigated system in routine shunt surgery can eliminate poor shunt placement resulting in a dramatic reduction in shunt revision rates.

\section{REFERENCES}

[1] Dandy WE, Blackfan KD. Internal hydrocephalus: an experimental, clinical and pathological study. Am J Dis Child 1914;8:112-6.

[2] Cho TH, Park JY, Lee JK, et al. Radiologic location of ventricular tip and the patency of ventriculoperitoneal shunt. Journal of Korean Neurosurgical Society 1997;26(4):513-7.

[3] Yamada SM, Kitagawa R, Teramoto A. Relationship of the location of the ventricular catheter tip and function of the ventriculoperitoneal shunt. J Clin Neuro Sci 2013;20(1):99-101.

[4] Pang D, Grabb PA. Accurate placement of coronal ventricular catheter using stereotactic co-ordinate guided free-hand passage. Technical note. J Neurosurg 1994;80(4):750-5.

[5] Bierbrauer KS, Storrs BB, McLone DG, et al. A prospective, randomized study of shunt function and infections as a function of shunt placement. Pediatr Neurosurg 1990-1991;16(6):287-91.
[6] Kestle JR, Drake JM, Cochrane DD, et al. Lack of benefit of endoscopic ventriculoperitoneal shunt insertion: a multicenter randomized trial. J Neurosurg 2003;98(2):284-90.

[7] Sekhar LN, Moossy J, Guthkelch AN. Malfunctioning ventriculoperitoneal shunts. Clinical and pathological features. J Neurosurg 1982;56(3):411-6.

[8] Tuli S, O'Hayon B, Drake J, et al. Change in ventricular size and effect of ventricular catheter placement in pediatric patients with shunted hydrocephalus. Neurosurgery 1999;45(6):1329-35.

[9] Lazareff JA, Peacock W, Holly L, et al. Multiple shunt failures: an analysis of relevant factors. Childs Nerv Syst 1998;14(6):271-5.

[10] Azeem SS, Origitano TC. Ventricular catheter placement with a frameless neuronavigational system: a 1-year experience. Neurosurgery 2007;60(4 Suppl 2):243-8.

[11] Wan KR, Toy JA, Wolfe R, et al. Factors affecting the accuracy of ventricular catheter placement. J Clin Neurosci 2011;18(4):485-8.

[12] Jung N, Kim D. Effect of electromagnetic navigated ventriculoperitoneal shunt placement on failure rates. J Korean Neurosurg Soc 2013;53(3):150-4.

[13] Price S, Santarius T, Richards H, et al. The accuracy of ventricular catheter placement: does it influence shunt revision rates? Cerebrospinal Fluid Research 2006;3(Suppl 1):S8.

[14] Kim YB, Lee JW, Lee KS, et al. Image-guided placement of ventricular shunt catheter. Journal of Clinical Neuroscience 2006;13(1):50-4. 